\title{
Cord compression: a rare complication of chronic lymphocytic leukaemia
}

\author{
G Majumdar, A K Singh
}

\begin{abstract}
Two years after the diagnosis, while the disease was under good control, a patient with chronic lymphocytic leukaemia (CLL) developed spinal cord compression from an extradural solid tumour composed of leukaemic cells. He was treated successfully with resection of the tumour followed by local radiotherapy and systemic chemotherapy.
\end{abstract}

Central nervous system complications, though common in acute leukaemias and in the blastic phase of chronic granulocytic leukaemia, are extremely rare in chronic lymphocytic leukaemia (CLL). ${ }^{12}$ We report a patient who developed acute cord compression as a result of extradural leukaemic deposit two years after CLL had been diagnosed.

\section{Case report}

A 66 year old man presented with cervical lumps and general malaise of three months' duration. Clinical examination showed generalised superficial lymphadenopathy and an enlarged spleen palpable $3 \mathrm{~cm}$ below the costal margin. There were no other abnormal physical signs. A blood count showed the following: haemoglobin $12.8 \mathrm{~g} / \mathrm{dl}$; a white cell count of $33.3 \times 10^{9} / 1$, with neutrophils $4.1 \times 10^{9} / 1$ and lymphocytes $28.0 \times 10^{9} / 1$; and platelets $197 \times 10^{9} / 1$. Liver and renal functions and serum immunoglobulins were normal. A chest $x$ ray picture showed hilar adenopathy but no parenchymal disease. Surface marker analysis of the peripheral blood lymphocytes showed HLA-DR $80 \%$, B-7 $70 \%$, but no surface immunoglobulins, and $\mathrm{T}$ cell markers in $<10 \%$. A bone marrow aspirate and trephine biopsy specimen showed dense infiltration by well differentiated small lymphocytes. B cell CLL was diagnosed.

The patient was treated for four months with chlorambucil, at a dose of $0.4 \mathrm{mg} / \mathrm{kg} /$ day for four days each month. He showed a satisfactory response, with disappearance of splenomegaly, regression of superficial lymphadenopathy, and a fall in his peripheral blood lymphocyte count to $6.0 \times 10^{9} / 1$. A repeat chest $x$ ray picture showed regression of hilar adenopathy. He was completely asymptomatic and no further treatment was given. One year after the diagnosis a transient $\operatorname{IgM}-\lambda$ paraprotein $(2-4 \mathrm{~g} / \mathrm{l})$ was noted on three occasions.
Twenty two months after the diagnosis of CLL he developed low back pain which rapidly got worse. Three days later he complained of weakness of his legs and on the next day developed paraplegia and paraesthesia of both legs with a clinically determined concentration of $L_{1}$. He had minimal superficial lymphadenopathy and no hepatosplenomegaly. Blood counts showed that the haemoglobin concentration was $13.4 \mathrm{~g} / \mathrm{dl}$, the white cell count $19.6 \times 10^{9} / 1$, with lymphocytes $15.7 \times 10^{9} / 1$, and a platelet count of $169 \times 10^{9} / 1$. A chest $x$ ray picture showed no progression of hilar adenopathy. A myelogram showed cord compression at the level of $T_{9-11}$ vertebrae. A specimen of cerebrospinal fluid showed raised protein $(1.2 \mathrm{~g} / \mathrm{l})$ concentration but a normal cell count. No abnormal cells were seen.

Emergency laminectomy and excision of the lesion, an extradural solid tumour $(6 \mathrm{~cm} \times$ $4 \mathrm{~cm}$ ), was carried out. Histological examination showed a homogeneous mass of well diffferentiated small lymphocytes with no suggestion of nodular configuration and fibrosis. The tumour cells had marker expression similar to that of peripheral blood lymphocytes. After surgery he received local radiotherapy and systemic chemotherapy with cyclophosphamide, vincristine, and prednisolone (CVP) for six months. His neurological deficits improved slowly and he was left with minimal residual paraparesis. His blood count at the end of chemotherapy was as follows: haemoglobin $12.9 \mathrm{~g} / \mathrm{dl}$; white cell count $6.5 \times 10^{9} / 1$, with lymphocytes $3.4 \times 10^{9} / 1$; and a platelet count of $141 \times 10^{9} / 1$. Residual superficial and hilar adenopathy persisted and he developed hypogammaglobulinaemia with a reduction in all three major classes of Ig. Over the next 12 months he had several bouts of chest infection. Blood counts and immuneparesis remained virtually unchanged. Three and a half years after the diagnosis of CLL he died of severe chest infection. Permission for necropsy was unfortunately refused.

\section{Discussion}

This patient had typical CLL at the time of diagnosis and responded well to a short course of chemotherapy. He remained asymptomatic and the disease remained under very good control without any further treatment until cord compression developed. Though the symptoms developed extremely rapidly, there was no evidence of progression or transforma-
Correspondence to:

Accepted for publication 31 July 1991 
tion to a more aggressive disorder like Richter's syndrome. In the only other reported case of cord compression caused by CLL the disease was also in the early stages at the time of this complication but the symptoms developed rather slowly-over three months. ${ }^{3}$ In both these cases the lesion was extradural, which is typical of a metastatic tumour. ${ }^{4}$

There have been several other reports of patients who had a more diffuse central nervous system disease as a result of blast cell infiltration of the brain or spinal cord. ${ }^{25-8}$ These patients had various symptoms like dementia, coma, and cerebral nerve palsy but no localised deposits. Post mortem examination in one series showed central nervous system infiltration by blast cells in 10 out of 14 cases of advanced CLL, though none of these patients had any neurological symptoms. ${ }^{9}$ The predominant lesion in one case of spinal cord disease was perivascular infiltration. ${ }^{8}$

Though cord compression is a very rare complication of CLL, it is potentially treatable and early diagnosis provides the best chance for the patient. Our patient showed surgically satisfactory improvement after surgical decompression followed by local radiotherapy. In the other reported case such measures could not be taken because of the delay in the diagnosis and because the disease rapidly proved fatal. ${ }^{3}$

1 Whitehouse JMA, Key HEM, eds. CNS complications of malignant diseases. London: MacMillan, 1975.

2 Hansen MM, Chronic lymphocytic leukaemia. Scand $J$ Haematol 1973;(Suppl 18):9-283.

3 Michalevicz R, Burstein A, Razon N, Reider I, Ilie B. Spinal epidural compression in chronic lymphocytic leukaemia. Cancer 1989;64:1961-4.

4 Constans JP, de Divitiis E, Donzelli R, Spaziante R, Melder JF, Haye C. Spinal metastasis with neurological manifestations: Review of 600 cases. J Neurosurg 1983;59:111-8.

5 Liepman MK, Votaw ML. Meningeal leukgemia tion chronic lymphocytic leukaemia. Cancer $1981 ; 47$. 2482-4.

6 Singh AK, Thompson RPH. Leukaemic meningitis in chronic lymphocytic leukaemia. Acta Haematol 1986;75:113-5.

7 Korsager S, Laursen B, Mortensen TM. Dementia and central nervous system involvement in chronic lymphocytic leukaemia. Scand J Haematol 1982;29:283-6.

8 Getaz EP, Miller GJ. Spinal cord involvement in chronic lymphocytic leukaemia. Cancer 1979;43:1858-61.

9 Reske-Nielsen E, Petersen JH, Sogaard H, Jensen KB. Leukaemia of the central nervous system. Lancet
1974;i:211-2.

\title{
Thorotrast granuloma: an unexpected diagnosis
}

\author{
S V Polacarz, R W Laing, R Loomes
}

\begin{abstract}
An example of a Thorotrast granuloma (thorotrastoma) occurred in the neck of a patient 44 years after a carotid angiogram in which Thorotrast was used as radiological contrast medium. The lesion had produced a "cold" abscess and the patient was undergoing treatment for retropharyngeal tuberculosis. Thorotrast leakage can produce unusual clinical symptoms and signs which are frequently misdiagnosed.
\end{abstract}

Between 1930 and 1950 as many as 100000 people were subjected to a large variety of radiological studies using the contrast medium Thorotrast, ${ }^{1}$ a colloidal suspension of radioactive thorium dioxide in a dextran medium. Due to its high density Thorotrast is well suited to its role as a radiological contrast medium, and was widely used in intravascular studies, particularly carotid angiography, and as luminal contrast in hollow body cavities, such as bladder and bronchus. Because Thorotrast is removed from the general circulation by reticuloendothelial macrophages, and hence concentrated in the liver, spleen and bone marrow, it was also used in radiological visualisation of these organs.
Thorotrast is, however, highly radioactive with predominant $\alpha$ emission and a half-life of $1.39 \times 10^{10}$ years. Excretion of ${ }^{232}$ thorium is negligible, and thus the body becomes the permanent site of deposition of this radioactive and potentially hazardous material. In spite of an increasing realisation of its carcinogenic potential ${ }^{2}$ Thorotrast continued to be used widely in many countries, including the United Kingdom until the early 1950 s.

\section{Case report}

A 66 year old man presented with a five week history of non-productive cough, sore throat, and severe pain in the right side of the neck and right occiput, with restricted neck movements and some dysphagia. He was treated, initially by his general practitioner, with antibiotics to no avail.

On subsequent examination in the ear, nose, and throat outpatients clinic the posterior pharyngeal wall on the right side was noted to be swollen with adherent inflammatory slough, and there was some induration of the right side of the neck. The palate was mobile and no abnormalities were detected in the nasal passages. Some fasciculation of the right side of the tongue was noted. Small lymph nodes were 